\title{
Exposure Assessment and Health Risk of Polybrominated Diphenyl Ether (PBDE) Flame Retardants in Indoor Environments of Children's Facilities in Korea
}

\author{
Ho-Hyun Kim, Ji-Yeon Yang, Yun-Suk Jang ${ }^{1)}$, Yong-Jin Lee, Chung-Soo Lee, Dong-Chun Shin ${ }^{2)}$ and \\ Young-Wook Lim* \\ Institute for Environmental Research, Yonsei University College of Medicine, 50 Yonsei-ro, Seodaemun-gu, Seoul 120-752, Korea \\ ${ }^{1)}$ Department of Environmental Engineering, Pohang University of Science and Technology, Pohang, Korea \\ ${ }^{2)}$ Department of Preventive Medicine, Yonsei University College of Medicine, 50 Yonsei-ro, Seodaemun-gu, Seoul 120-752, Korea \\ *Corresponding author. Tel: +82-2-2228-1898, E-mail: envlim@yuhs.ac
}

\begin{abstract}
This study assessed the health risks of childhood exposure to PBDEs via different possible pathways in children's facilities and indoor playgrounds. When PBDE contamination was measured, it was determined through multiple routes, including inhalation of indoor dust, dermal contact with product surfaces and children's hands, and incidental dust ingestion. Samples were collected from various children's facilities (playrooms, daycare centers, kindergartens, and indoor playgrounds) during summer (Jul-Sep, 2007) and winter (Jan-Feb, 2008). The hazard index (HI) was estimated for non-carcinogens, and PBDEs, such as TeBDE, PeBDE, HxBDE, and DeBDE, were examined. The sensitivity to the compounds did not exceed $1.0(\mathrm{HI})$ for any of the subjects in any facility. However, current data about toxicity does not reflect effects that were fully sensitive in children, so there is uncertainty in the dose-response data. The contribution rates of PBDEs were 71.4 to $96.1 \%$ and 3.7 to $28.2 \%$ for intake and inhalation exposure, respectively, indicating that intake of floor dust and inhalation are the primary routes.
\end{abstract}

Key words: PBDEs, Children's facilities, Risk assessment, Multi-route exposure

\section{INTRODUCTION}

Poly-brominated diphenyl ethers (PBDEs) are well known semi-volatile organic compounds (SVOCs) that have been widely used as flame retardants (Umweltbundesamt, 2000). PBDEs are economical and effective compounds used for their flame retardant properties in various products such as electronic goods and curtains.
Their toxicity of these compounds has been studied since they were first detected in human bodies in the 2000s, and global regulations have been established (US EPA, 2008a, b). Commercial mixtures of PBDEs are marketed as PentaBDE, OctaBDE, and DecaBDE. All three types have been linked to hepatic toxicity, endocrine disorders, neurodevelopmental disorders, and development and reproductive disorders (Birnbaum and Cohen Hubal, 2006). According to the Korean Ministry of Gender Equality \& Family (MOGEF) concerning flame retardant products in childcare facilities, there is a requirement that flame resistant products should be installed in childcare-facilities. Although this regulation is meant to protect people from fire, the products become additional sources of PBDEs as "Endocrine Disrupting Chemicals (EDCs)" (Birnbaum and Staskal, 2004). An EDC is best defined as "an exogenous substance that causes adverse health effects in an intact organism or its progeny, secondary to changes in endocrine function" (EEC, 1996). It has been reported that children incidentally ingest more dust than adults, and that this may be their primary route of exposure to PBDEs (Stapleton et al., 2005; Webster et al., 2005).

PBDEs are manufactured in three commercial forms - penta, octa, and deca. The penta commercial product is primarily used in polyurethane foams, in carpet underlays, vehicle interiors, furniture and microprocessor packaging in computers. The octa-product (along with other flame retardants) can be used to treat thermoplastics such as high impact polystyrene and acrylonitrile-butadiene-styrene copolymers. The deca-product is used in plastic housings for electrical goods such as TVs, computers, and textiles (Weil and Levchik, 2007; Hazrati and Harrad, 2006).

However, studies on PBDEs and their risk in children's facilities have rarely been conducted in South 
Korea, and it is difficult to determine the differences between harmful effects caused by chronic and acute exposures. Therefore, this needs to be evaluated through a Health Risk Assessment (HRA). For PBDEs without any established environmental standards, an HRA is one of the methods used to quantitatively assess the effects of chronic exposure via multiple pathways (Health Canada, 2004).

Therefore, this study aims to investigate exposure pathways of PBDEs as EDCs from products in major places of activity for children such as childcare facilities and indoor playgrounds and to determine their total risk levels through multiple exposure routes using HRA.

\section{MATERIALS AND METHODS}

\subsection{Selection of Major Places of Activity for Children and Contents of Survey}

Indoor playgrounds and childcare facilities (playrooms, daycare centers, and kindergartens) were investigated for this study. We chose 20 playrooms, 20 daycare centers, 20 kindergartens and 20 indoor-playgrounds located in three cities around Korea (Seoul, Busan and Yeosu). As a reference, we used 2007 data from 33,499 childcare centers and 8,294 kindergartens countrywide. We chose these three cities to represent metropolitan, small and large-sized cities, and industrial complexes, respectively. We chose newly-built facilities both small and large in size. This research did not include childcare centers run by enterprises or by $\mathrm{pu}-$ blic authorities.

The first investigation measurements, performed in summer and conducted with the cooperation of the facilities, were conducted in July, August, and September 2007. The second investigation, performed twice in winter, was related to exposure assessment and was conducted in January and February 2008. In the first and second investigations, the same facilities were repeatedly investigated. Investigations were conducted in the main play and rest areas of indoor facilities. Researchers chose a space where study and play were conducted in parallel to the representative point based on individual classes in the case of childcare facilities.

Since the composition of floor dust can change according to time and place, accumulated dust was evaluated in many different spots in order to reduce error. Wipe samples of representative items in the facilities were examined once in the winter survey (the second investigation) from January to February 2008. In the three regions, two to three representative items (a desk, a chair, and a floor mat) from each of the four types of facility (indoor playground, playroom, daycare center, and kindergarten) were selected for surface wipe samples.

Representative spaces and items in a facility were determined based on the questionnaire survey of the directors, teachers and business owners. Among items in representative spaces, those with a high frequency of contact and a high utility rate for education and play were ranked, and the top four were designated as representative items.

This study quantitatively examined four types of PBDEs - TeBDE, PeBDE, HxBDE and DeBDE. The major exposure pathways examined in this study were floor dust, indoor air, surface wipe and hand wipe.

\section{2 Indoor Air Sample}

Our method for analyzing the presence of PBDEs was based on Method 1614 (US EPA, 2007). PBDE samples from indoor air were prepared by collecting particulate and gaseous air with a high volume air sampler equipped with a filter and passive air samplers (PAS) consisting of a polyurethane foam disk (PUF, Tisch Environmental, Inc; certified "flame retardant" free) housed in a stainless steel domed chamber. Air sampling methods were performed using the same method as in Allen et al. (2007). The PUF was washed with methylene chloride (MC) and dried completely before sample collection. It was covered with foil, stored in a zipper bag and then placed in a desiccator. The filter was placed in an oven at $450^{\circ} \mathrm{C}$ for one day before use. The weight of the filter was gauged before and after sample collection, and the total suspended particle (TSP) mass was determined based on this difference. Each filter was sealed in a zipper bag after sample collection. To pretreat the collected sample, each PUF and filter were placed in a Soxhlet extractor, and the sample was condensed to $1 \mathrm{~mL}$ with a BUCHI rotavapor after a 20-hour extraction using 300 $\mathrm{mL}$ of $\mathrm{MC}$ as a solvent. The concentrated sample was loaded into a column packed with multi silica gel and refined using $150 \mathrm{~mL}$ of hexane. It was then condensed to $1 \mathrm{~mL}$ with a BUCHI rotavapor. The concentrated sample was subjected to gel permeation chromatography (GPC) and condensed to $1 \mathrm{~mL}$ using $30 \mathrm{~mL}$ of MX/ hexane (1:1) after discarding $15.5 \mathrm{~mL}$ of $\mathrm{MX} /$ hexane. The concentrated sample was placed in a GC vial, and the remaining sample was completely evaporated using a nitrogen evaporator. Completely pretreated air samples were analyzed by the gas chromatograph (GC)/ high resolution mass spectrometer (HRMS) HP 6890 series GC system, Agilent/ JMS-700T, JEOL.

\section{3 Indoor Dust Sample}

We used a standardized collection protocol for dust (Colt et al., 1998). Researchers collected dust samples 
from the living room, classroom and playroom areas during both sampling rounds. The living room, classroom and playroom areas were selected as the rooms where the children spent most of their time and which had a high likelihood of containing products with PBDEs. Dust samples were collected with a vacuum cleaner. A vacuum bag was used for each sample, and one dust sample per facility was obtained by vacuuming all floor coverings.

For dust samples of PBDEs, the collected dust was sifted with a $500 \mu \mathrm{m}$ sieve to remove impurities. After weighing an empty sample paper, $1 \mathrm{~g}$ of the dust was moved onto the paper. After quantitatively measuring the weight before pretreatment, it was placed in a 50 $\mathrm{mL}$ vial to soak sufficiently in dichloromethane (DCM), with ultrasonic extraction being performed twice an hour. Acidification using a high concentration of sulfuric acid was repeated until the hexane layer was colorless. The concentrated sample was loaded into a column packed with multi silica gel and refined using $150 \mathrm{~mL}$ of hexane. This was condensed to $1 \mathrm{~mL}$ with a BUCHI rotavapor. The concentrated sample was subjected to gel permeation chromatography (GPC) and refined by spilling $1: 1 \mathrm{MC} /$ hexane. This was condensed again to $1 \mathrm{~mL}$ with a BUCHI rotavapor, placed in a GC vial and then completely evaporated using a nitrogen evaporator. Conditions of pretreatment and analysis instruments were the same as those of the air samples. The analytical methodology is as described above as for the air samples.

\section{4 Surface Wipe Sample}

Methodology for the surface wipe collections was adapted from Gordon et al. (1999). Cotton brand gauze pads $(7.6 \mathrm{~cm} \times 7.6 \mathrm{~cm})$ were used to collect product surface wipes. A template was used to ensure that a uniform surface area of $10 \mathrm{~cm} \times 10 \mathrm{~cm}\left(100 \mathrm{~cm}^{2}\right)$ was sampled. The time since the surface was cleaned, wiped or otherwise touched prior to this study could not be determined (Toms et al., 2009). Surface wipes were collected from desks, chairs, mats and televisions in the same rooms from which the air and dust samples were taken.

For surface wipe samples of PBDEs, cotton gauze pads were washed with DCM before use and completely dried so as not to have remaining washing solvent on the gauze (cotton gauze: Johnson and Johnson SofWick). Before sample collection, the cotton gauze was washed with MC and dried completely. It was covered with foil, sealed in a zipper bag and placed in a desiccator. After soaking the cotton gauze pads in $4 \mathrm{~mL}$ of isopropanol with a wet cloth, the surfaces of the items were wiped 4-5 times with the pads. The pads were put in brown vials and stored in a freezer. As pretreat- ment, a surface wipe sample was placed in a Soxhlet extractor and extracted with $300 \mathrm{~mL}$ of $\mathrm{MC}$ for 20 hours. After extraction, it was concentrated to $1 \mathrm{~mL}$ with a BUCHI rotavapor, and the concentrated sample was loaded into a column packed with multi silica gel and refined using $150 \mathrm{~mL}$ of hexane. This was condensed to $1 \mathrm{~mL}$ with a BUCHI rotavapor. The condensed sample was subjected to GPC and concentrated to 1 $\mathrm{mL}$ with a BUCHI rotavapor by discarding $15.5 \mathrm{~mL}$ of $1: 1 \mathrm{MC} /$ hexane and removing $30 \mathrm{~mL}$ of sample. This was concentrated to $1 \mathrm{~mL}$ with an evaporator, and the condensed sample was placed in a GC vial. After evaporating the remaining sample with a nitrogen evaporator, it was analyzed with a GC/HRMS HP 6890 series GC system, Agilent/JMS-700T, JEOL.

\section{5 Hand Wipe Sample}

Methodology for hand wipe collection was adapted from Gordon et al. (1999). Cotton brand gauze pads $(7.6 \mathrm{~cm} \times 7.6 \mathrm{~cm})$ were immersed in $3 \mathrm{~mL}$ of isopropyl alcohol in a clean aluminum foil tray. All samples were collected by wiping the entire surface area of a child's hands, top and bottom, including the sides of the hands and fingers.

To obtain hand wipe samples of PBDEs, the cotton gauze pads were washed with DCM before its use and completely dried to remove any washing solvent (cotton gauze: Johnson and Johnson Sof-Wick). Both hands were wiped with cotton gauze pads $(7.5 \mathrm{~cm} \times 7.5 \mathrm{~cm})$ soaked in $4 \mathrm{~mL}$ isopropanol. The pads were placed in brown glass vials and stored in a freezer. Conditions of pretreatment and analysis instruments were the same as those of the surface wipe samples. The analytical methodology was as described above for the surface wipe samples.

\section{6 QA/QC}

To secure the recovery rate of each isomer of PBDEs, 13C substituents (13C-BDE-28, -47, -99, -100, -153, $-154,-183,-209)$ were spiked before pretreatment of the samples. The limit of detection (LOD) varied by isomer, especially BDE-47, BDE-99 and BDE-209, whose rates were high in the environment showing relatively high LODs. Relative standard deviations (RSD) of the relative sensitivity factors (RRF) of the calibration curve met the conditions of $\pm 15 \%$ or less. LODs by route and material are presented in Table 1 . Recovery rates of PBDEs by pathway were slightly different: $61-79 \%$ in the air, $76-85 \%$ in dust, $83-117 \%$ in surface samples and $75-111 \%$ in wipe samples.

\section{7 Exposure Patterns}

The facility and other general information were investigated through a questionnaire and interview form 
filled out by the nursery teacher. Factor value deduction to calculate the extent of exposure and investigation of exposure configuration analysis were performed by separating the subjects into four age groups. A total of 16 young children were investigated: playroom (6 months to 2 years), daycare center ( 2 to 5 years), kindergarten (5 to 6 years), and indoor playground (3 to 9 years). The interview included general questions related to the use of childcare facilities and indoor playgrounds, characteristics of the children's play (time and frequency of play by days of the week, time, and other), and information about the children.

To observe the specific exposure mode in the case of the nurseries, researchers filmed 40 minutes of free playtime with the cooperation of the facilities. To de-

Table 1. Estimated limits of detection for PBDEs in multimedia samples.

\begin{tabular}{lrrr}
\hline & $\begin{array}{c}\text { Indoor air } \\
\left(\mu \mathrm{g} / \mathrm{m}^{3}\right)\end{array}$ & $\begin{array}{c}\text { Dust } \\
(\mu \mathrm{g} / \mathrm{g})\end{array}$ & $\begin{array}{c}\text { Surface wipe/ } \\
\text { Hand-wipe }\left(\mu \mathrm{g} / \mathrm{cm}^{2}\right)\end{array}$ \\
\hline BDE 28 & 64.50 & 45.12 & 43.97 \\
BDE 47 & 3222.93 & 110.56 & 1158.90 \\
BDE 100 & 561.52 & 15.05 & 152.56 \\
BDE 99 & 2129.27 & 57.72 & 628.49 \\
BDE 154 & 123.45 & 14.01 & 12.50 \\
BDE 153 & 108.74 & 21.68 & 105.67 \\
BDE 183 & 148.41 & 25.35 & 35.36 \\
BDE 209 & 3500.81 & 309.78 & 409.18 \\
\hline
\end{tabular}

termine exposure patterns through investigation and to examine specific exposure patterns, 40 minutes of voluntary playtime were observed and recorded after securing approval of the childcare facilities. The staff advised us that the children were most active and their play was most varied during voluntary playtime, although there were differences according to age, so we mainly observed voluntary playtime in the morning. Forms of play included standing, sitting, walking, running, and rolling around (Table 2).

Children can be exposed to PBDEs by inhaling semivolatile organic pollutants from indoor products. Skin exposure can occur through contact with various surfaces. Routes of oral intake exposure are hand to mouth through hands contaminated by PBDEs and from the ingestion of dust from the floor.

\section{8 Dose-response Data}

Toxicity according to material is presented in Table 3. Classification of levels of danger was conducted based on toxic effects through infant and childhood period exposure and then based on carcinogenesis, genital organ toxicity, growth toxicity, nerve toxicity, immune toxicity, and next generation toxicity. Based on the data review, we used the following to categorize the materials: carcinogenicity for those that could potentially provoke cancer, non-carcinogenicity for those producing toxicity according to amount regardless of

Table 2. Exposure factors of children based on video and direct observation.

\begin{tabular}{|c|c|c|c|c|}
\hline \multirow[b]{2}{*}{ Site } & \multicolumn{4}{|c|}{ Factor } \\
\hline & $\begin{array}{l}\text { Age group } \\
\text { (years) }\end{array}$ & Activity type & Exposure routes & Specificity \\
\hline Play room & $0.5-2$ & Sitting/Lying & $\begin{array}{l}\text { Inhalation, Dermal (object), } \\
\text { Ingestion (dust, hand) }\end{array}$ & $\begin{array}{c}\text { Mean time spent } \\
5-10 \mathrm{hrs} / \text { day } \\
\text { (frequency of activity time 2-7) }\end{array}$ \\
\hline \multirow{3}{*}{ Daycare center } & 2 & $\begin{array}{c}\text { Sitting/Lying/ } \\
\text { Standing/Walking }\end{array}$ & $\begin{array}{l}\text { Inhalation, Dermal (object), } \\
\text { Ingestion (dust, hand) }\end{array}$ & \multirow{3}{*}{$\begin{array}{c}\text { Mean time spent } \\
5-10 \mathrm{hrs} / \text { day } \\
\text { (frequency of activity time 5-7) } \\
\text { *sleeping/meal time } \\
\text { average time } 3\end{array}$} \\
\hline & $3-4$ & $\begin{array}{l}\text { Sitting/Lying/ } \\
\text { Standing/Walking/ } \\
\text { Running }\end{array}$ & $\begin{array}{l}\text { Inhalation, Dermal (object), } \\
\text { Ingestion (dust, hand) }\end{array}$ & \\
\hline & 5 & $\begin{array}{c}\text { Sitting/Lying } \\
\text { Standing/Walking/ } \\
\text { Running }\end{array}$ & $\begin{array}{l}\text { Inhalation, Dermal (object), } \\
\text { Ingestion (dust, hand) }\end{array}$ & \\
\hline Kindergarten & $6-7$ & $\begin{array}{c}\text { Sitting/Lying } \\
\text { Standing/Walking/ } \\
\text { Running }\end{array}$ & $\begin{array}{l}\text { Inhalation, Dermal (object), } \\
\text { Ingestion (dust, hand) }\end{array}$ & $\begin{array}{l}\text { Mean time spent } \\
5 \mathrm{hrs} / \text { day } \\
\text { *except sleeping }\end{array}$ \\
\hline \multirow{2}{*}{$\begin{array}{l}\text { Indoor playground } \\
\text { (fees charged) }\end{array}$} & $3-4$ & $\begin{array}{c}\text { Sitting/Lying } \\
\text { Standing/Walking, } \\
\text { Running/Wallow }\end{array}$ & $\begin{array}{l}\text { Inhalation, Dermal (object), } \\
\text { Ingestion (dust, hand) }\end{array}$ & $\begin{array}{l}\text { Weekday time spent } \\
1-2 \text { time/wk }\end{array}$ \\
\hline & $5-9$ & $\begin{array}{c}\text { Sitting/Lying } \\
\text { Standing/Walking, } \\
\text { Running/Wallow }\end{array}$ & $\begin{array}{l}\text { Inhalation, Dermal (object), } \\
\text { Ingestion (dust, hand) }\end{array}$ & $\begin{array}{c}\text { Weekday time spent } \\
1-2 \text { time/wk, } \\
\text { except exposure hand-to-mouth }\end{array}$ \\
\hline
\end{tabular}


Table 3. Dose-response assessment of PBDEs.

\begin{tabular}{|c|c|c|c|c|c|c|c|}
\hline Compound & $\begin{array}{l}\text { Classification } \\
\text { of material }\end{array}$ & $\begin{array}{l}\text { Exposure } \\
\text { route }\end{array}$ & Endpoint & $\begin{array}{c}\text { BMDL or } \\
\text { NOAEL } \\
\text { (mg/kg-day) }\end{array}$ & UF & $\begin{array}{c}\text { RfD }(C) \\
(\mathrm{mg} / \mathrm{kg} \text {-day) }\end{array}$ & Reference \\
\hline $\begin{array}{l}\text { Tetrabromodiphenyl } \\
\text { ether (TeBDE) }\end{array}$ & $\begin{array}{c}\text { Non } \\
\text { Carcinogenic }\end{array}$ & All & $\begin{array}{c}\text { Neurobehavioral } \\
\text { effects }\end{array}$ & 0.35 & 3000 & $1.0 \times 10^{-4}$ & $\begin{array}{l}\text { Eriksson } \text { et al. (2001) } \\
\text { US EPA IRIS (2008d) }\end{array}$ \\
\hline $\begin{array}{l}\text { Pentabromodiphenyl } \\
\text { ether(PeBDE) }\end{array}$ & $\begin{array}{c}\text { Non } \\
\text { Carcinogenic }\end{array}$ & All & $\begin{array}{c}\text { Neurobehavioral } \\
\text { effects }\end{array}$ & 0.29 & 3000 & $1.0 \times 10^{-4}$ & $\begin{array}{l}\text { Viberg et al. }(2004 a) \\
\text { US EPA IRIS (2008c) }\end{array}$ \\
\hline $\begin{array}{l}\text { Hexabromodiphenyl } \\
\text { ether (HxBDE) }\end{array}$ & $\begin{array}{c}\text { Non } \\
\text { Carcinogenic }\end{array}$ & All & $\begin{array}{c}\text { Neurobehavioral } \\
\text { effects }\end{array}$ & 0.45 & 3000 & $2.0 \times 10^{-4}$ & $\begin{array}{c}\text { Viberg (2003a) } \\
\text { US EPA IRIS (2008b) }\end{array}$ \\
\hline $\begin{array}{l}\text { Decabromodiphenyl } \\
\text { ether(DeBDE) }\end{array}$ & $\begin{array}{c}\text { Non } \\
\text { Carcinogenic }\end{array}$ & All & $\begin{array}{l}\text { Neurobehavioral } \\
\text { effects }\end{array}$ & 2.22 & 300 & $7.0 \times 10^{-3}$ & $\begin{array}{c}\text { Viberg }(2003 b) \\
\text { US EPA IRIS (2008a) }\end{array}$ \\
\hline
\end{tabular}

exposure time, and EDC for those that caused sensitivities in children such as endocrine disorders. Doseresponse data for HRA were determined by collecting and reviewing reports on toxicity officially suggested by the Integrated Risk Information System (IRIS) of the US EPA and World Health Organization (WHO).

\subsection{Application of Exposure Factors and Formula Following Establishment of Exposure Scenario}

Exposure factors used in the formula to calculate the extent of exposure were determined by comparing and assessing values obtained through literature review, questionnaires, observations and actual measurements. Exposure factors identified in each exposure equation are defined in Table 4. Body weight for age groups 0.52, 3-4, 5-6 and 7-9 years are derived from the Korean Exposure Factors Handbook (KEFH) (Jang et al., 2007) and are 10, 16, 20 and $30 \mathrm{~kg}$, respectively. Body surface area for age groups $0.5-2,3-4,5-6$ and 7-9 years are derived from the KEFH (Jang et al., 2007) and are $4,352,6,567,7,914$ and $9,896 \mathrm{~cm}^{2}$, respectively. The ratio of hand to mouth versus inhalation and ingestion rates is derived from the US Child-specific Exposure Factors Handbook (US CEFH) (US EPA, 2002) and the KEFH (Jang et al., 2007). The definition of sucking was derived from the National Institute of Environmental Research (NIER, 2007) and Zartarian (2005). Exposure period and time were derived from the KEFH (Jang et al., 2007) and from this study via questionnaire and measurement.

Representative formulas (1), (2) (3) and (4) are defined according to exposure pathway, and the grounds for the values used in the formulas are presented in Table 4.

$$
\begin{aligned}
& \mathrm{LADD}_{\text {inh,air,e }}=\mathrm{C}_{\text {indooraire }} \times \mathrm{IR}_{\text {inh }} \times \mathrm{T}_{\mathrm{e}} \\
& \mathrm{LADD}_{\text {inh,aire, }}: \text { Inhalation exposure dose in indoor air } \\
& \quad(\mathrm{mg} / \mathrm{kg} / \mathrm{day}) \\
& \mathrm{C}_{\text {indoorair,e }} \text { Indoor air concentration at facility }\left(\mathrm{mg} / \mathrm{m}^{3}\right)
\end{aligned}
$$

$\mathrm{IR}_{\mathrm{inh}}$ : Inhalation rate per body weight for exposure scenario $\left(\mathrm{m}^{3} / \mathrm{hr}-\mathrm{kg}\right)$

$\mathrm{T}_{\mathrm{e}}$ : Exposure time adjustment for exposure scenario $\left(=\mathrm{ED}_{\mathrm{j}} \times \mathrm{EF}_{\mathrm{e}} \times \mathrm{ET}_{\mathrm{e}} / \mathrm{AT}\right)($ unitless $)$

$\mathrm{LADD}_{\text {ingest,dust } \mathrm{e}}=\mathrm{C}_{\text {dust } \mathrm{e}} \times \mathrm{IR}_{\text {dust }} \times \mathrm{T}_{\mathrm{e}}$

$\mathrm{LADD}_{\text {ingest,_HtM }, \mathrm{e}}=\left(\mathrm{C}_{\text {prod.e }} \times \mathrm{SA}_{\text {prod. }} \times \mathrm{MN}_{\text {prod. }}\right.$ $\left.\times\left(\mathrm{MT}_{\text {prod. }} / 3600\right)\right) \times \mathrm{T}_{\mathrm{e}}$

$\mathrm{LADD}_{\text {ingest,dust,e }}$ : Ingestion exposure dose of house dust ( $\mathrm{mg} / \mathrm{kg} /$ day)

$\mathrm{LADD}_{\text {ingest,_HtM,e: }}$ Ingestion exposure by hand-tomouth or toy-to-mouth $(\mathrm{mg} / \mathrm{kg} /$ day $)$

$\mathrm{C}_{\text {dust,e }}$ : Concentration of indoor dust at facility $(\mathrm{mg} /$ kg-dust)

$\mathrm{C}_{\text {prod.e.: }}$ Concentration of pollutant on surface of product (hand, floor, toy, etc.) $\left(\mathrm{mg} / \mathrm{cm}^{2}\right)$

$\mathrm{IR}_{\text {dust }}$ : Ingestion rate per body weight of subject $(\mathrm{kg}$ dust/day-kg)

$\mathrm{SA}_{\text {prod: }}$ Surface area of the product (hand, floor, toy, etc.) that is being mouthed $\left(\mathrm{cm}^{2}\right)$

$\mathrm{MN}_{\text {prod. }}$ : Number of mouthings per hour for the product (hand, floor, toy, etc.) (times/hr)

$\mathrm{MT}_{\text {prod: }}$ : Exposure times per mouthing for the product (hand, floor, toy, etc.) (sec/time)

$\mathrm{T}_{\mathrm{e}}$ : Exposure time adjustment for exposure scenario (unitless)

$\mathrm{LADD}_{\text {dermal,res,j.k, }}=\mathrm{C}_{\text {surf,k }} \times \mathrm{SA}_{\mathrm{j}} \times \mathrm{FA}_{\mathrm{k}} \times \mathrm{CT}_{\text {contact, } \mathrm{j}, \mathrm{k}}$

$$
\times \mathrm{T}_{\mathrm{e}} \times \mathrm{TF}_{\text {surf-skin,k }}
$$

LADD $_{\text {dermal,res,j,k,e: }}$ Dermal exposure dose of interior product (mg/kg/day)

$\mathrm{C}_{\text {surf, } \mathrm{k}}$ : Concentration of product surface, $\mathrm{k}$, at facility $\left(\mathrm{mg} / \mathrm{cm}^{2}\right)$

$\mathrm{SA}_{\mathrm{j}}$ : Surface area of subject per body weight $\left(\mathrm{cm}^{2} /\right.$ $\mathrm{kg}$ )

$\mathrm{FA}_{\mathrm{k}}$ : Fraction of exposed skin, $\mathrm{k}$, to surface area (unitless)

$\mathrm{CT}_{\mathrm{k}, \mathrm{e}}$ : Contact time of dermal contact per day by product, $\mathrm{k}$ (hrs/day) 


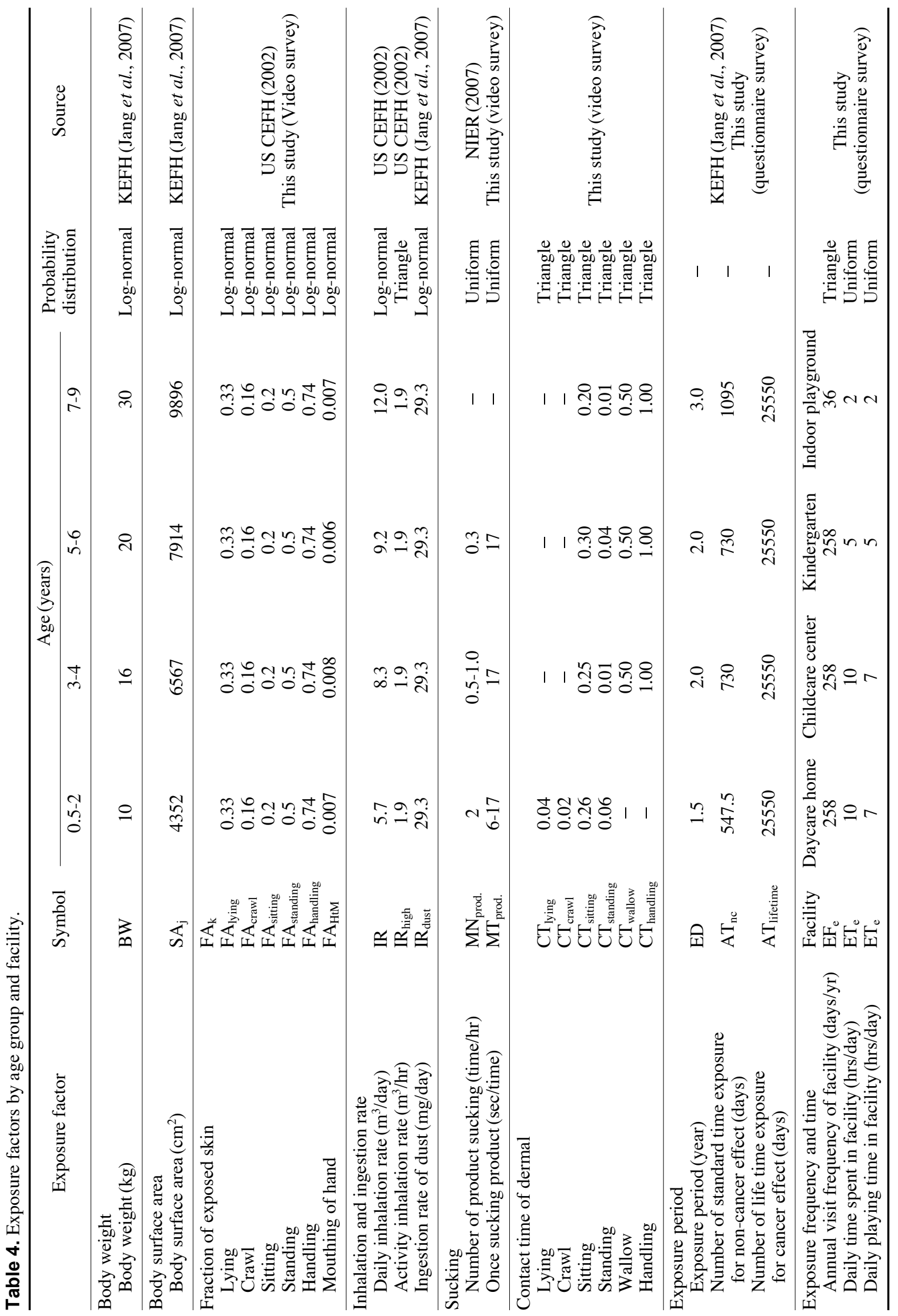


$\mathrm{TF}_{\text {surf-skin,k }}$ : Transfer factor from product to exposed skin (unitless)

$\mathrm{T}_{\mathrm{e}}$ : Exposure time adjustment for exposure scenario (unitless)

\subsection{Risk Calculation}

In this study, the target groups were infants (6 months-2 years old), toddlers (3-4 years old), preschool children (5-6 years old), and school children below the age of 10 (7-9 years old). Data on the quantitative toxicity of carcinogenic and non-carcinogenic materials for dose-response assessment were primarily collected from studies on exposure of young children; if there was no data for children, data on exposure in adults were used. For EDC, a Tolerable Daily Intake (TDI) was decided, and if there was no TDI, it was replaced with a Reference dose (RfD) to calculate the Hazard Index (HI). Final health risk probability distribution values were obtained using the probability distribution values of LADD according to the type of facility, material and age. Health risk assessment was performed using the values of $50 \%$ and $95 \%$ for health risk probability distributions and the criteria of 0.1 to 1 for $\mathrm{HI}$ and HQ, respectively.

$$
\begin{aligned}
& \mathrm{HQ}_{\mathrm{r}, \mathrm{p}, \mathrm{i}}=\mathrm{LADD}_{\mathrm{r}, \mathrm{p}, \mathrm{i}} / \mathrm{RfD}_{\mathrm{r}, \mathrm{p}} \\
& \mathrm{HI}_{\mathrm{i}}=\sum \mathrm{HQ}_{\mathrm{r}, \mathrm{p}, \mathrm{i}} \\
& \mathrm{HQ}_{\mathrm{r}, \mathrm{p}, \mathrm{i}}: \text { Hazard quotient via exposure route } \mathrm{r} \text { of pol- } \\
& \quad \text { lutant } \mathrm{p} \text { at age } \mathrm{i} \text {. } \\
& \mathrm{HI}_{\mathrm{i}}: \text { Hazard index via multi-route at age } \mathrm{i} \text { for com- } \\
& \quad \text { plex mixture pollutant such as PBDEs } \\
& \mathrm{LADD}_{\mathrm{r}, \mathrm{p}, \mathrm{i}}: \text { Lifetime Average Daily Dose via expo- } \\
& \quad \text { sure route } \mathrm{r} \text { of pollutant } \mathrm{p} \text { at age } \mathrm{i}(\mathrm{mg} / \mathrm{kg} / \mathrm{day}) \text {. } \\
& \mathrm{RfD}_{\mathrm{r}, \mathrm{p}}: \text { Reference dose per day via exposure route } \mathrm{r} \\
& \quad \text { of pollutant } \mathrm{p}(\mathrm{mg} / \mathrm{kg} / \text { day }) \text {. }
\end{aligned}
$$

\subsection{Statistical Analysis}

To assess exposure to PBDEs (Penta-, Octa-, and Deca-BDE) per medium, the suggested average, minimum, and maximum values, comparison of the target child facilities and the majority group medium, along with the statistical significance were assessed through nonparametric analysis. In air, dust and wipe samples, all congeners were non-normally distributed. Two-tailed, Pearson and Spearman correlation coefficients between variables were determined. Statistical analysis was performed using SPSS 16.0 for Windows.

\section{RESULTS AND DISCUSSION}

\section{1 Multi-route Exposure Assessment}

The assessment of multi-route exposure of PBDEs is presented in Table 5. When indoor air samples were collected, concentrations were less than $1,440 \mathrm{pg} / \mathrm{m}^{3}$ for indoor playgrounds, less than $1,021 \mathrm{pg} / \mathrm{m}^{3}$ for kindergartens, $789 \mathrm{pg} / \mathrm{m}^{3}$ for daycare centers, and $366 \mathrm{pg} /$ $\mathrm{m}^{3}$ for playrooms. Detection rates were $100 \%$. High concentrations in indoor playgrounds may be a result of the majority of them being located in large shopping marts and open to exposure to various sources of electronic equipment and wood-based materials as well as flame-proof textiles (Umweltbundesamt, 2000) and consumer products (Allen et al., 2008). Zhao (2004) insisted that PBDEs in air accumulated in chairs and carpets including polyurethane foam and could be emitted into the air. In other words, polyurethane foam chairs and carpets were both primary and secondary contamination sources (Umweltbundesamt, 2000).

Congener profiles for indoor air in children's facilities were dominated by BDE-209, followed by BDE47. This was consistent with findings from other studies (Cetin and Odabasi, 2011; Toms et al., 2009). A previous study in Australia (Toms et al., 2009) reported that levels were $0.5-179 \mathrm{pg} / \mathrm{m}^{3}$ and $15-486 \mathrm{pg} / \mathrm{m}^{3}$ at homes and in offices, respectively, which were lower than those in the facilities for children in this study. Toms et al. (2009), Mandalakis et al. (2008), and Gevao et al. (2006) showed that recorded levels of PBDEs ranged from 12 to $2,247 \mathrm{pg} / \mathrm{m}^{3}$ in indoor air (Table 6). In contrast to public buildings, average PBDE concentrations in the indoor air of Athens (Mandalakis et al., 2008) were higher than the average concentrations in this study.

Overall PBDE concentrations in dust samples ranged from 560 to $32,835 \mathrm{ng} / \mathrm{g}$. Indoor playgrounds were more heavily contaminated with PBDEs (mean 16,901 ng/g) than other children's facilities (means: daycare center $8,334 \mathrm{ng} / \mathrm{g}>$ kindergarten $6,158 \mathrm{ng} / \mathrm{g}$ and $>$ playroom $1,556 \mathrm{ng} / \mathrm{g}$ ), which may be due to the ensemble of complex indoor sources as mentioned above (PBDEs-air). According to studies by Kang et al. (2011), Toms et al. (2009), Allen et al. (2008) and Harrad et al. (2008), concentrations were notably different by country (Table 7). Our study indicated that PBDE contamination in indoor playgrounds was similar to that of electronic factories found by Kang et al. (2011). Toms et al. (2009) reported concentrations of $86-3,070 \mathrm{ng} / \mathrm{g}$ in indoor dust at homes and offices, which was lower than for the facilities for children in this study. In this exploratory assessment, overall building characteristics did not appear to affect PBDE dust concentrations, which was in agreement with other studies (Tan et al., 2007; Stapleton et al., 2005; Wilford et al., 2005). PBDE concentrations at children's facilities were generally higher than those found in homes or offices, except in a Birmingham, UK study (Harrad et al., 2008). Indoor dust exposure occurs through a variety of pathways 
Table 5. Concentrations of PBDEs at children's facilities.

\begin{tabular}{|c|c|c|c|c|c|c|c|c|c|c|c|c|c|}
\hline \multirow[t]{2}{*}{ Site } & \multirow[t]{2}{*}{ Compound } & \multicolumn{3}{|c|}{ Indoor air $\left(\mathrm{pg} / \mathrm{m}^{3}\right)$} & \multicolumn{3}{|c|}{ Floor dust (ng/g) } & \multicolumn{3}{|c|}{$\begin{array}{l}\text { Product wipe surface } \\
\qquad\left(\mathrm{pg} / \mathrm{cm}^{2}\right)\end{array}$} & \multicolumn{3}{|c|}{$\begin{array}{l}\text { Hand wipe surface } \\
\qquad\left(\mathrm{pg} / \mathrm{cm}^{2}\right)\end{array}$} \\
\hline & & $\mathrm{n}$ & Mean & Range & $\mathrm{n}$ & Mean & Range & $\mathrm{n}$ & Mean & Range & $\mathrm{n}$ & Mean & Range \\
\hline \multirow{11}{*}{ Play room } & pentaBDE & & & & & & & & & & & & \\
\hline & BDE28 & & 8.75 & $1.36-24.96$ & & 18.60 & $<$ LOD-55.81 & & 0.46 & $<$ LOD-1.40 & & 0.04 & $<$ LOD-0.19 \\
\hline & BDE47 & & 220.34 & $<$ LOD-1111.6 & & 45.21 & $5.79-118.03$ & & 17.29 & $<$ LOD-62.89 & & $<\mathrm{LOD}$ & - \\
\hline & BDE99 & & 76.08 & $<$ LOD-387.5 & & 23.73 & $8.59-50.91$ & & 11.40 & $<$ LOD-31.02 & & $<\mathrm{LOD}$ & - \\
\hline & BDE100 & & 32.52 & $<$ LOD-163.9 & & 3.48 & $1.38-6.35$ & & 2.26 & $<$ LOD-8.24 & & $<\mathrm{LOD}$ & - \\
\hline & BDE153 & & 1.92 & $<$ LOD-7.47 & & 26.87 & $17.30-44.30$ & & 1.71 & $<$ LOD-10.23 & & 0.86 & $<$ LOD-4.31 \\
\hline & BDE154 & & 2.93 & $<$ LOD-13.99 & & 5.58 & $2.93-10.07$ & & 0.76 & $<$ LOD-3.39 & & 0.14 & $0.08-0.19$ \\
\hline & octaBDE & & & & & & & & & & & & \\
\hline & $\begin{array}{l}\text { BDE183 } \\
\text { decaBDE }\end{array}$ & & 1.36 & $<$ LOD-6.61 & & 125.96 & $105.21-165.94$ & & 4.46 & $0.07-29.93$ & & 5.90 & $0.29-25.09$ \\
\hline & BDE209 & & 22.90 & $<$ LOD-84.92 & & 1307.15 & $705.36-2328.69$ & & 185.6 & $3.97-1153.56$ & & 28.25 & $22.68-42.02$ \\
\hline & Total PBDEs & 20 & 366.80 & $6.87-1793.98$ & 6 & 1556.59 & $861.06-2780.12$ & 6 & 224.0 & $4.07-1206.38$ & 6 & 35.20 & $23.08-52.26$ \\
\hline \multirow{11}{*}{$\begin{array}{l}\text { Daycare } \\
\text { center }\end{array}$} & pentaBDE & & & & & & & & & & & & \\
\hline & BDE28 & & 12.66 & $0.47-66.58$ & & 0.82 & $<$ LOD-2.93 & & 0.01 & $<$ LOD-0.03 & & $<\mathrm{LOD}$ & - \\
\hline & BDE47 & & 427.23 & $1.25-3374.10$ & & 77.03 & $5.82-260.10$ & & 1.65 & $<$ LOD-5.29 & & $<\mathrm{LOD}$ & - \\
\hline & BDE99 & & 159.42 & $0.95-1425.99$ & & 100.64 & $7.35-342.63$ & & 2.50 & $<$ LOD-7.94 & & $<\mathrm{LOD}$ & - \\
\hline & BDE100 & & 64.43 & $0.2-578.54$ & & 17.81 & $1.19-61.62$ & & 0.41 & $<$ LOD-1.26 & & $<\mathrm{LOD}$ & - \\
\hline & BDE153 & & 1.05 & $<$ LOD-5.17 & & 31.52 & $3.01-98.49$ & & 2.94 & $<$ LOD- 13.48 & & 0.20 & $<$ LOD-0.81 \\
\hline & BDE154 & & 4.46 & $<$ LOD-35.17 & & 18.37 & $1.02-63.97$ & & 0.39 & $0.04-1.12$ & & 0.11 & $<$ LOD-0.24 \\
\hline & BDE183 & & 1.85 & $<$ LOD-10.94 & & 345.63 & $13.71-1177.25$ & & 24.31 & $0.11-119.15$ & & 3.36 & $0.28-12.02$ \\
\hline & decaBDE & & & & & & & & & & & & \\
\hline & BDE209 & & 118.11 & $<$ LOD-1095.9 & & 7742.29 & $525.44-26607.30$ & & 566.2 & $4.40-1936.81$ & & 26.70 & $11.43-44.06$ \\
\hline & Total PBDEs & 20 & 789.21 & $4.99-5490.78$ & 6 & 8334.11 & $560.95-28614.29$ & 6 & 598.4 & $5.07-2070.55$ & 6 & 30.37 & $12.31-57.12$ \\
\hline \multirow{10}{*}{$\begin{array}{l}\text { Kinder- } \\
\text { garten }\end{array}$} & pentaBDE & & & & & & & & & & & & \\
\hline & BDE28 & & 8.03 & $0.33-77.12$ & & $<$ LOD & - & & 0.02 & $<$ LOD-0.13 & & $<\mathrm{LOD}$ & - \\
\hline & BDE47 & & 278.85 & $<$ LOD-4818.8 & & 7.13 & $2.85-13.82$ & & 1.03 & $<$ LOD-6.17 & & $<\mathrm{LOD}$ & - \\
\hline & BDE99 & & 87.78 & $<$ LOD-1593.71 & & 8.79 & $4.93-13.59$ & & 0.95 & $<$ LOD-5.70 & & 0.45 & $<$ LOD-2.25 \\
\hline & BDE100 & & 35.56 & $<$ LOD-667.54 & & 1.79 & $0.78-3.48$ & & 0.18 & $<$ LOD-1.10 & & 0.08 & $<$ LOD-0.38 \\
\hline & BDE153 & & 2.52 & $<$ LOD-29.78 & & 43.25 & $7.25-68.58$ & & 0.11 & $<$ LOD-0.66 & & $<\mathrm{LOD}$ & - \\
\hline & $\begin{array}{l}\text { BDE154 } \\
\text { octaBDE }\end{array}$ & & 3.70 & $<$ LOD-63.79 & & 6.54 & $1.31-10.40$ & & 0.08 & $<$ LOD-0.37 & & 0.08 & $<$ LOD-0.20 \\
\hline & $\begin{array}{l}\text { BDE183 } \\
\text { decaBDE }\end{array}$ & & 3.08 & $<$ LOD-15.33 & & 259.45 & $37.14-409.37$ & & 0.55 & $<$ LOD-2.36 & & 0.19 & $<$ LOD-0.41 \\
\hline & BDE209 & & 601.49 & $<$ LOD-11727.8 & & 5831.09 & $1899.10-12102.8$ & & 61.31 & $<$ LOD-259.3 & & 16.36 & $0.12-27.76$ \\
\hline & Total PBDEs & 20 & 1021.00 & $4.50-12000.88$ & 6 & 6158.03 & $1953.35-12604.8$ & 6 & 64.23 & $<$ LOD-275.8 & 6 & 17.15 & $0.12-30.35$ \\
\hline \multirow{11}{*}{$\begin{array}{c}\text { Indoor } \\
\text { playground }\end{array}$} & pentaBDE & & & & & & & & & & & & \\
\hline & BDE28 & & 16.31 & 0.85-59.99 & & 26.15 & $<$ LOD-58.30 & & 0.01 & $<$ LOD-0.03 & & $<\mathrm{LOD}$ & - \\
\hline & BDE47 & & 590.06 & $<$ LOD-4530.78 & & 103.57 & $3.25-209.88$ & & 0.28 & $<$ LOD-1.39 & & $<\mathrm{LOD}$ & - \\
\hline & BDE99 & & 294.40 & $0.58-1962.86$ & & 48.52 & $6.62-96.43$ & & 0.52 & $<$ LOD-1.90 & & $<\mathrm{LOD}$ & - \\
\hline & BDE100 & & 95.69 & $<$ LOD-729.57 & & 5.09 & $1.00-15.17$ & & 0.06 & $<$ LOD- 0.28 & & $<\mathrm{LOD}$ & - \\
\hline & BDE153 & & 7.31 & $0.10-30.30$ & & 43.05 & $8.39-83.72$ & & 0.21 & $<$ LOD- 0.83 & & 0.14 & $<$ LOD-0.27 \\
\hline & BDE154 & & 10.80 & $0.07-81.03$ & & 14.32 & $1.75-34.90$ & & 0.11 & $0.04-0.31$ & & 0.09 & $<$ LOD-0.13 \\
\hline & octaBDE & & & & & & & & & & & & \\
\hline & $\begin{array}{l}\text { BDE183 } \\
\text { decaBDE }\end{array}$ & & 10.38 & $0.21-122.97$ & & 331.58 & $40.75-628.4$ & & 0.99 & $0.13-3.25$ & & 1.66 & $1.13-3.20$ \\
\hline & BDE209 & & 415.38 & $9.12-3574.28$ & & 16329.6 & $912.27-31757.0$ & & 93.12 & $35.20-203.88$ & & 385.67 & $27.20-456.12$ \\
\hline & Total PBDEs & 20 & 1440.33 & $23.44-7510.77$ & 6 & 16901.9 & $974.03-32835.8$ & 6 & 95.29 & $35.58-208.29$ & 6 & 387.56 & $273.42-453.41$ \\
\hline
\end{tabular}

including consumption of dust-contaminated food and respiration of dust (Butte et al., 2002; Lioy et al., 2002).

Out of the eight isomers, BDE-209 accounted for over $90 \%$ of the total concentration of PBDEs. Previ- ous studies by Kang et al. (2011), Toms et al. (2009), Allen et al. (2008) and Harrad et al. (2008) corroborate this result. Stapleton and Dodder (2008) recently reported the evidence of photodegradation of BDE-209 in house dust under controlled exposure to sunlight. 
Table 6. Summary of concentrations $\left(\mathrm{pg} \mathrm{m}^{-3}\right)$ of PBDE congeners in indoor air $\left(\mathrm{pg} \mathrm{m}^{-3}\right)$ samples from this and other studies.

\begin{tabular}{|c|c|c|c|c|c|}
\hline Location & Site & $\begin{array}{l}\text { Statistical } \\
\text { parameter }\end{array}$ & $\begin{array}{c}\text { Congener } \\
209\end{array}$ & $\sum \mathrm{BDE}$ & Reference \\
\hline Seoul etc, Korea ${ }^{a}$ & $\begin{array}{l}\text { Children's facilities } \\
\qquad(\mathrm{n}=80)\end{array}$ & Average & $22-601$ & $366-1440$ & This study \\
\hline Izmir, Turkey ${ }^{b}$ & $\begin{array}{c}\text { Offices, laboratories \& } \\
\text { Homes }(n=15)\end{array}$ & Arithmetic mean & $39-168$ & $43.5-206$ & Cetin and Odabasi, 2011 \\
\hline Queensland, Australia ${ }^{c}$ & $\begin{array}{c}\text { Home }(n=6) \& \\
\text { Office }(n=3)\end{array}$ & Average & $<3.7-117$ & $0.5-486$ & Toms et al., 2009 \\
\hline Brisbane, Australia $^{\mathrm{d}}$ & Home $(n=10)$ & Average & n.a. & $21-341$ & Toms et al., 2009 \\
\hline \multirow{4}{*}{ Athens, Greece ${ }^{\mathrm{e}}$} & $\begin{array}{l}\text { Internet Cafes \& } \\
\text { PC rooms }(\mathrm{N}=7)\end{array}$ & Average & n.a. & 174 & \multirow{4}{*}{ Mandalakis et al., 2008} \\
\hline & $\begin{array}{l}\text { Public build. \& } \\
\text { Offices }(N=5)\end{array}$ & Average & n.a. & 2247 & \\
\hline & $\begin{array}{l}\text { PC \& Electr. } \\
\text { Shops }(N=5)\end{array}$ & Average & n.a. & 117 & \\
\hline & $\begin{array}{l}\text { Furniture stores } \\
\qquad(\mathrm{N}=2)\end{array}$ & Average & n.a. & 12 & \\
\hline Kuwait $^{\mathrm{f}}$ & $\begin{array}{l}\text { Home }(n=46) / \\
\text { Office }(n=24)\end{array}$ & Average & n.a. & $15.2-32.7$ & Gevao et al., 2006 \\
\hline
\end{tabular}

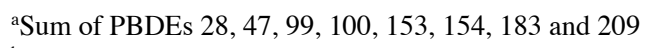

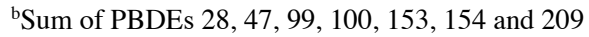

${ }^{\mathrm{c} S u m}$ of PBDEs 47, 99, 100, 153, 154, 183 and 209

${ }^{\mathrm{d}}$ Sum of PBDEs 47, 99, 100, 153, 183 and 209

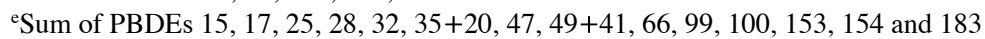

${ }^{\mathrm{f}}$ Sum of PBDEs 28, 47, 99, 100, 153, 154 and 183

n.a. not applicable.

Detection rates of BDE-28 and others were $35 \%$ and $100 \%$, respectively.

Fig. 2 presents scatterplots of air versus dust concentrations for pentaBDE and decaBDE in the children's facilities. This study also found that indoor air and dust had no significant association with pentaBDE $\left(\mathrm{r}^{2}=0.074, \mathrm{p}=0.273\right)$ in the main living area, similar to Allen et al. (2008). However, Wilford et al. (2005) found significant correlations between PBDE concentrations in the air and dust collected from the same homes. The difference may be due to the higher volatility of pentaBDE, which allows partitioning between vapor, suspended particulate and dust (Allen et al., 2008).

When wipe samples from childcare facility objects such as TVs, mats, computers and monitors were analyzed, no PBDEs were detected ( $<$ LOD to $2,070.55$ $\mathrm{pg} / \mathrm{cm}^{2}$ ). Results of the current study were slightly lower than those of the surface wipes from four computers/monitors in the USA (Schecter et al., 2005). Like airborne dust, BDE-209 accounted for $90 \%$ of the total concentration in surface wipe samples. Since deca-BDE has been used for housings in electronic devices, it can be emitted from the surface of TVs and computers. In addition, deca-BDE can be found in contaminated dust on the surfaces of those products. Because BDE-209 exists mainly in particulate materials rather than gaseous ones, removing dust on the surface of products and floors is important to environmental public health and reducing exposure to BDE209.

Hand wipe samples yielded 0.12 to $453 \mathrm{pg} / \mathrm{cm}^{2}$ of PBDEs, with the highest concentration found in indoorplaygrounds, $387 \mathrm{pg} / \mathrm{cm}^{2}$. This is likely due to children engaging in more physical activity when playing and increased contact with game machines and other children in indoor playgrounds than in other childcare facilities. Hand wipe samples also showed the highest detection rate of BDE-209 at 90\%, but the level was lower than surface wipe samples. A previous study by Heather et al. (2008) gathered hand wipe samples by wiping both hands of 16 males and 17 females including six children aged 8 to 10 years and found concentrations ranging from 3.3 to $1,970 \mathrm{pg} / \mathrm{cm}^{2}$.

Based on reported concentrations of PBDEs in indoor air, house dust, interior products and toys, $50^{\text {th }}$ percentile and $95^{\text {th }}$ percentile levels of average daily dose (ADD) of total PBDEs ranged from $1.32^{-4}$ to 
Table 7. Summary of concentrations $\left(\mathrm{ng} \mathrm{g}^{-1}\right)$ of PBDE congeners in dust samples from this and other studies.

\begin{tabular}{|c|c|c|c|c|c|}
\hline Location & Site & $\begin{array}{l}\text { Statistical } \\
\text { parameter }\end{array}$ & $\begin{array}{c}\text { Congener } \\
209\end{array}$ & $\sum \mathrm{BDE}$ & Reference \\
\hline Seoul etc, Korea ${ }^{a}$ & Children's facilities $(n=24)$ & Average & $1307-16329$ & $1556-16901$ & This study \\
\hline \multirow{6}{*}{ Hong Kong, China ${ }^{\mathrm{b}}$} & Commercial offices $(n=20)$ & \multirow{6}{*}{ Average } & $51.1^{\mathrm{g}}$ & 5620 & \multirow{6}{*}{ Kang et al., 2011} \\
\hline & Secondary schools $(n=4)$ & & $61.2^{\mathrm{g}}$ & 4780 & \\
\hline & Shopping malls $(n=5)$ & & $61.5^{\mathrm{g}}$ & 7230 & \\
\hline & Hospitals $(n=16)$ & & $52.1^{\mathrm{g}}$ & 4940 & \\
\hline & Electronic factories $(n=6)$ & & $72.5^{\mathrm{g}}$ & 14400 & \\
\hline & Manufacturing plants $(n=4)$ & & $47.1^{\mathrm{g}}$ & 5970 & \\
\hline Queensland, Australia $^{c}$ & Home $(n=6) /$ Office $(n=3)$ & Average & $37-587 / 100-2230$ & $86-733 / 583-3070$ & Toms et al., 2009 \\
\hline Boston, US ${ }^{\mathrm{d}}$ & Home $(n=108)$ & Geometric mean & $1703^{\mathrm{h}} / 4502^{\mathrm{i}}$ & $6255^{\mathrm{h}} / 13732^{\mathrm{i}}$ & Allen et al., 2008 \\
\hline Toronto, Canada ${ }^{\mathrm{e}}$ & Home $(n=20)$ & Average & 670 & 1400 & Harrad et al., 2008 \\
\hline Birmingham, UK $^{\mathrm{f}}$ & Home $(n=28)$ & Average & 45000 & 45000 & Harrad et al., 2008 \\
\hline
\end{tabular}

a Sum of PBDEs 28, 47, 99, 100, 153, 154, 183 and 209

${ }^{\mathrm{b}}$ Sum of PBDEs 3, 7, 15, 17, 28, 47, 49, 66, 71, 77, 85, 99, 100, 119, 126, 138, 153, 154, 156, 184, 183, 191, 196, 197, 207, 206 and 209

'Sum of PBDEs 47, 99, 100, 153, 154, 183 and 209

${ }^{\mathrm{d}}$ Sum of PBDEs 17, 28/33, 47, 49, 66, 75, 85/155, 99, 100, 138, 153, 154, 183, 196, 197, 203, 206, 207, 208 and 209

eSum of PBDEs 28, 47, 49, 66, 99, 100, 153, 154, 183, 196, 197, 203 and 209

fSum of PBDEs 28, 47, 49, 66, 99, 100, 153, 154, 183, 196, 197, 203 and 209

${ }^{\mathrm{g} B D E}-209$ contributed the greatest proportion to total PBDEs in dust $(\%)$

hedroom

${ }^{\mathrm{i}}$ Main living room

$7.46 \times 10^{-4} \mu \mathrm{g} / \mathrm{kg}$-day and $1.50 \times 10^{-3}$ to $1.64 \times 10^{-2}$ $\mu \mathrm{g} / \mathrm{kg}$-day, respectively, for various age groups of children. The $50^{\text {th }}$ percentile ADDs of total PBDEs by age group were $6.80 \times 10^{-4} \mu \mathrm{g} / \mathrm{kg}$-day for 0.5 to 2 -yearolds in a play-room, $7.46 \times 10^{-4} \mu \mathrm{g} / \mathrm{kg}$-day for 3-4year-olds in a daycare center, $4.28 \times 10^{-4} \mu \mathrm{g} / \mathrm{kg}$-day for 5 to 6-year-olds in a kindergarten, and $1.32 \times 10^{-4}$ $\mu \mathrm{g} / \mathrm{kg}$-day for 3 to 4 -year-olds in an indoor playground. The $95^{\text {th }}$ percentile ADDs of total PBDEs by age groups were $0.003 \mu \mathrm{g} / \mathrm{kg}$-day for 0.5 to 2 -year-olds in a play-room, $0.016 \mu \mathrm{g} / \mathrm{kg}$-day for 3 to 4 -year-olds in a daycare center, $0.006 \mu \mathrm{g} / \mathrm{kg}$-day for 5 to 6 -year-olds in a kindergarten, and $0.002 \mu \mathrm{g} / \mathrm{kg}$-day for 3 to 4 -yearolds in an indoor playgrounds. The ADD of 3 to 4year-olds in a daycare center was the highest. The major human exposure pathway was ingestion (above $90 \%$ of total ADD) of house dust and mouthing for all age groups and facilities. For Canadian research of PBDEs, the intake via house dust and indoor air of PBDEs for 0 to 6-month-old infants, 0.5 to 4-yearolds, 5 to 11-year-olds, 12 to 19 -year-olds, and adults were $0.23 \mu \mathrm{g} / \mathrm{kg}$-day, $0.36 \mu \mathrm{g} / \mathrm{kg}$-day, $0.12 \mu \mathrm{g} / \mathrm{kg}$-day, $0.028 \mu \mathrm{g} / \mathrm{kg}$-day, and $0.024 \mu \mathrm{g} / \mathrm{kg}$-day, respectively. The age group with the greatest potential exposure via indoor air and dust was 0 to 11-year-olds (Health Canada, 2004). The ADDs in the present study were lower than those in the Canadian children research because the Canadian study used an upper-bounding estimation approach based on reported concentrations of PBDEs in Canada for screening assessments.

\section{2 Health Risk Assessment}

Hazard index (HI) was calculated by synthesizing exposure factors. Exposure scenarios for PBDEs are presented in Table 8.

Taking into consideration the physiological and behavioral characteristics of children in the facilities, the HI values of non-carcinogenic toxicity of PBDEs were evaluated. No material or facility had an HI greater than 0.1. Fiftieth percentile (median) HIs of exposure to PBDEs were $0.0007,0.001,0.0004$ and less than 0.0001 in playrooms, daycare centers, kindergartens and indoor playgrounds, respectively. Ninety-fifth percentile HIs were $0.003,0.01,0.002$ and 0.002 in playrooms, daycare centers, kindergartens and indoor playgrounds, respectively.

Ingestion, inhalation and skin PBDE exposure rates were $94.0 \%, 5.9 \%$ and $0.1 \%$, respectively, in play rooms. Hence, exposure through the intake of floor dust was the primary route (indoor dust $79.8 \%$, indoor air $20 \%$, hand wipe $0.1 \%$, and surface wipe $0.1 \%$ ). Daycare centers (indoor dust $78.0 \%$, indoor air $21.6 \%$, hand wipe $0.3 \%$, and surface wipe $0.1 \%$ ) and kindergartens (indoor dust $71.4 \%$, indoor air $28.2 \%$, hand wipe $0.3 \%$, and surface wipe $0.1 \%$ ) had similar results. However, as in indoor playgrounds (those charging admission fees), the recorded rates of indoor dust, indoor air, hand wipe and surface wipe exposure were 

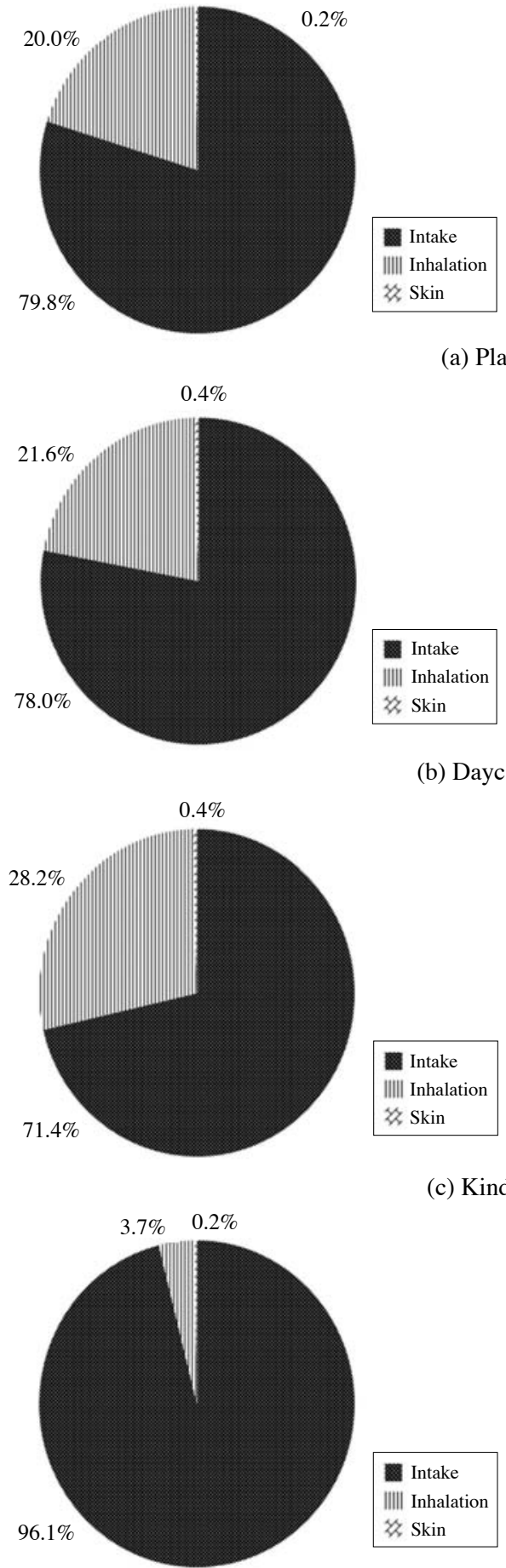
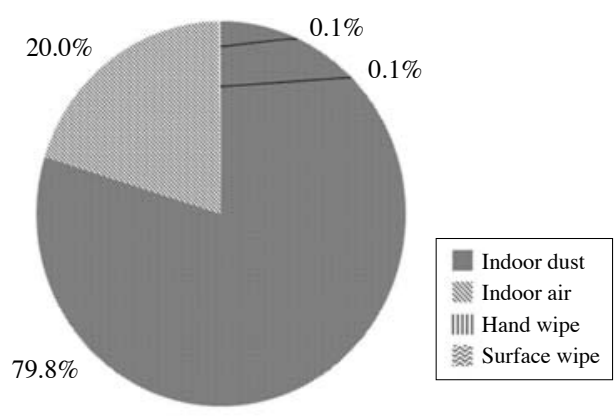

(a) Play rooms
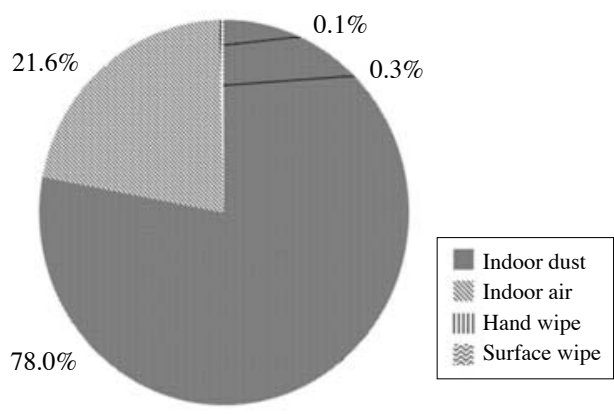

(b) Daycare centers
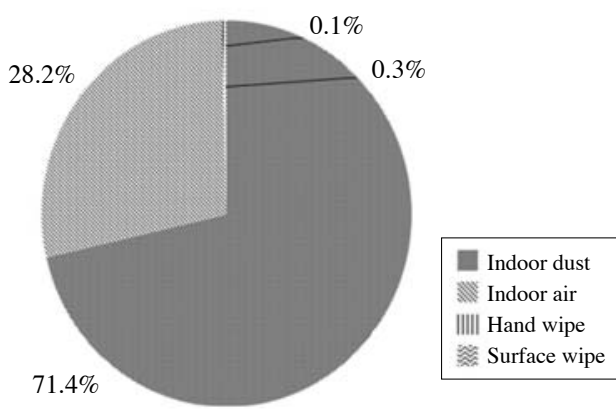

(c) Kindergartens

(d) Indoor playgrounds (fees charged)

Fig. 1. Contribution rates according to exposure route of PBDEs.

$96.1 \%, 3.7 \%, 0.1 \%$ and $0.1 \%$, respectively. Ingestion of floor dust was the primary exposure route (Fig. 1). We considered many causes for this difference from the other settings. Based on a survey regarding the number and type of potentially PBDE-treated items (e.g., chairs, sofas, TVs, and other electronic items), we cannot identify an obvious cause of these very high concentrations. The median HI for all children's faci- 

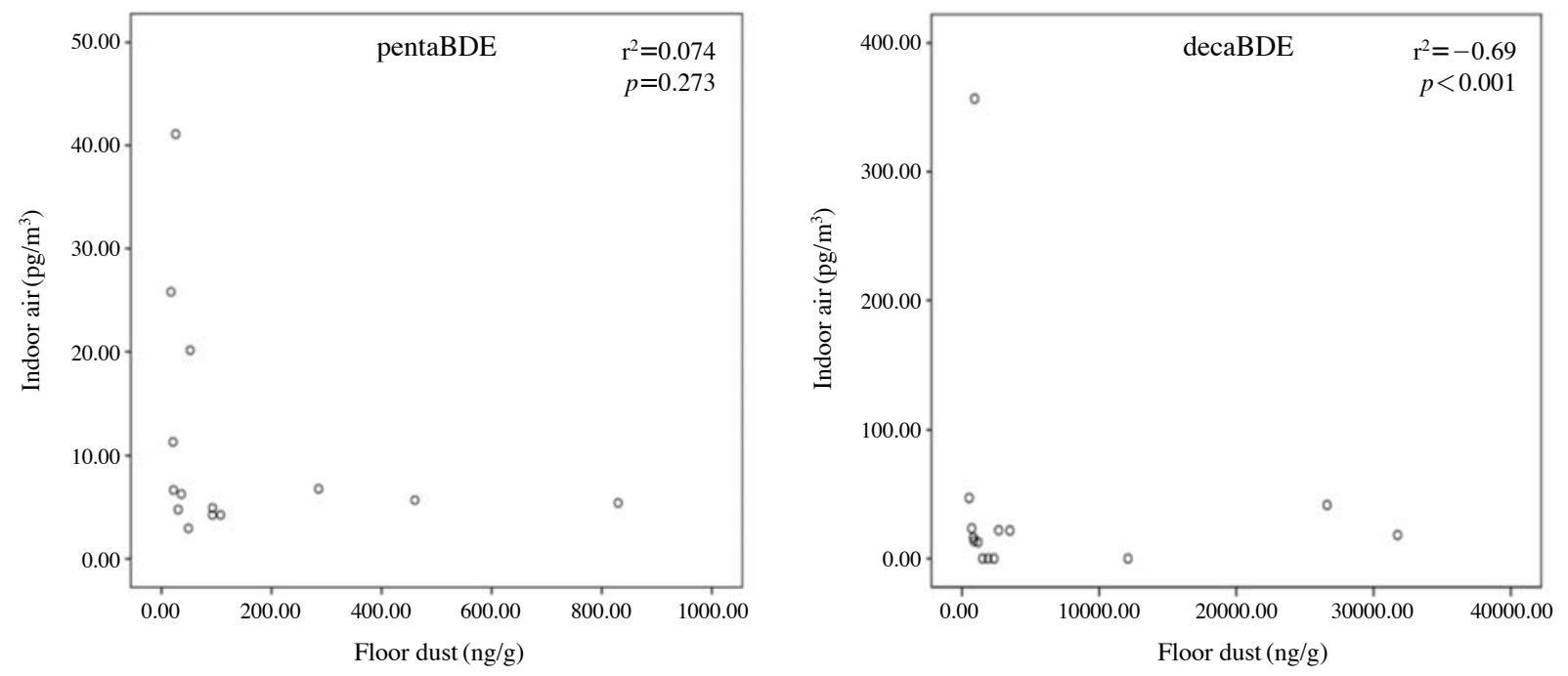

Fig. 2. Correlations between PBDE congeners in air $\left(\mathrm{ng} / \mathrm{m}^{3}\right)$ and dust (ng/g).

lities for 0.5 to 6 -year-olds through multi-route was 0.006 (Table 8 ), and the rate of contribution of $\mathrm{HI}$ in daycare centers was greater than $75 \%$ (Fig. 3).

Analyses of exposure to young children where the potential for exposure is high require further consideration. Because young children spend large amounts of time in these facilities ( 6 to 10 hours) and are exposed to various consumer products (e.g., floor mats, carpets, curtains, furniture, television and electronic devices) by various pathways (sucking, rolling and dust ingestion), their risks are relatively high in playrooms and daycare centers. Allen et al. (2008) suggest that microenvironments play an important role in the distribution of pentaBDE and decaBDE in dust. This lack of uniformity was expected since PBDEs are thought to have sources that are specific to children's facilities. As kindergartens hours decrease (five hours on average) and more time is devoted to education with low activity levels, the risk of exposure decreases. The risk of indoor playgrounds also decreases if they are used for fewer hours (1-2 hours) and less frequently (36 times per year on average).

Health Canada (2004) conducted and published the 'State of the Science Report for a Screening Health Assessment of PBDEs by the Canadian Environmental Protection Act.' Total intakes (ambient air, indoor air, house dust, drinking water, soil, and food) of PBDEs for 0-6-month-old infants, 0.5 to 4-year-olds, 5 to 11-year-olds, 12 to 19 -year-olds, and adults in Canada were $2.6 \mu \mathrm{g} / \mathrm{kg}$-day, $0.95 \mu \mathrm{g} / \mathrm{kg}$-day, 0.6 $\mu \mathrm{g} / \mathrm{kg}$-day, $0.3 \mu \mathrm{g} / \mathrm{kg}$-day, and $0.28 \mu \mathrm{g} / \mathrm{kg}$-day, respectively, in this report. The potentially highest exposure route for the 0 to 6 months age group was breastfeed- ing ( $92 \%$ of total intake), while that for 0.5 to 11 -yearolds (children) was food (69\% of total intake) and house dust ( $31 \%$ of total intake). The health risk (HI) of the most highly exposed age group (0 to 6 month infants) was approximately 0.003 (Health Canada, 2004). Although these results were assessed under conservative conditions considering the selected critical effect level and deterministic estimates of exposure, consistent with the preliminary nature of screening health assessments, the conservative health risk does not take into account the potential continuing increase in body burden of PBDEs (based on data for the most highly exposed age group) should similar use patterns continue. Our results are similar to the levels in the Canadian report. To determine the adequacy of PBDE risk assessment for addressing elements of uncertainty associated with limitations of the database for health effects and population exposure (in which confidence overall is considered to be moderate), intraspecies and interspecies variations in sensitivity, extrapolation from acute exposure to chronic exposure for the critical effect, and biological adversity or severity of the effects requires additional in-depth evaluation of the relevant data and development of additional, more meaningful information on population exposure to PBDEs.

Our assessment of risk based on actual measured data had some shortcomings. Residents were not continuously exposed to the same concentrations of PBDEs in the indoor air during their period of residence. We were also unable to monitor lifetime exposure.

In this study, we investigated 20 facilities in three cities, so the study population did not fully represent the general population. Although this study examined 

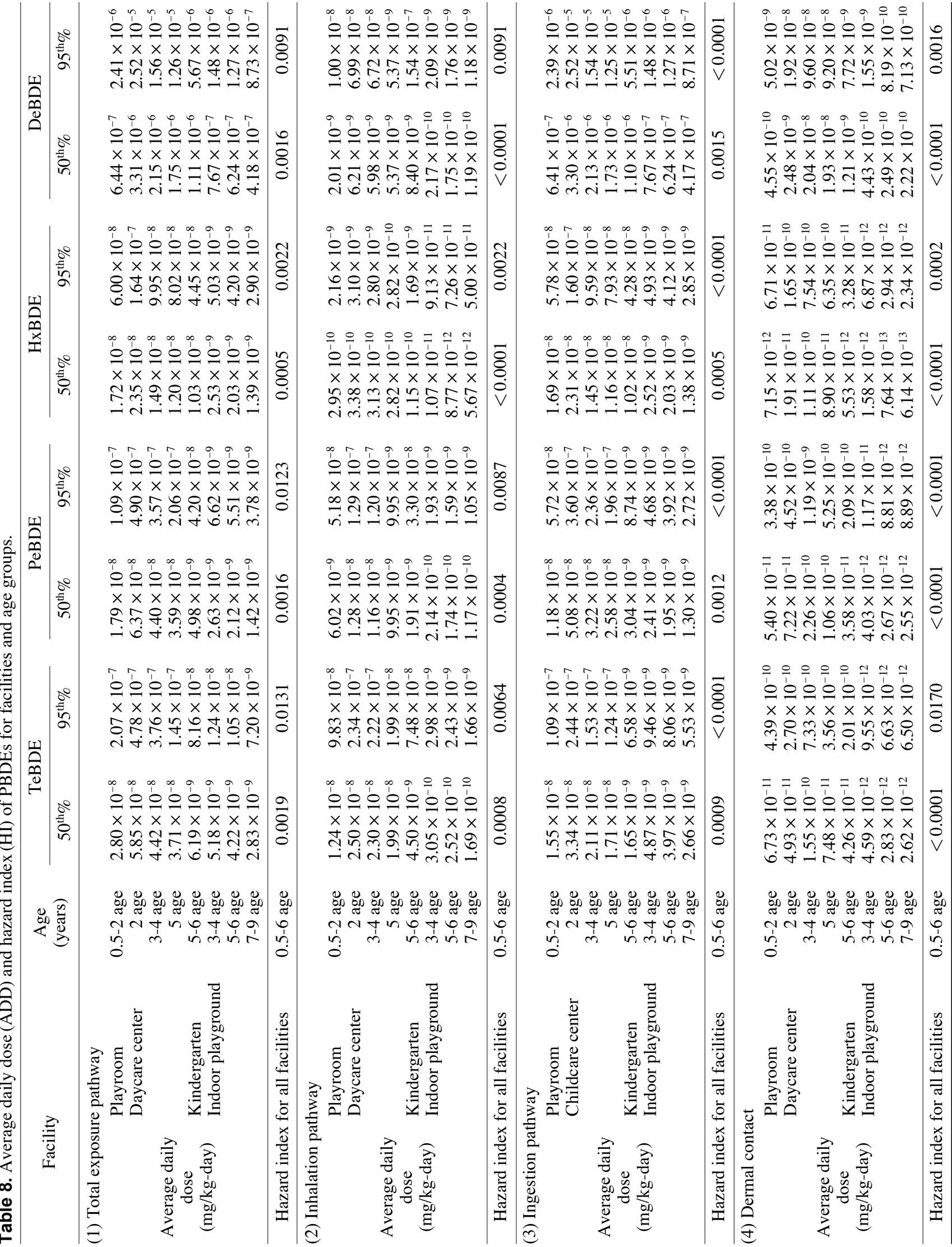

$=00 \div=\simeq \simeq \simeq$ 으으으으으으으음

$\times \times \times \times \times \times \times$

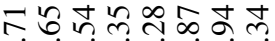

o-

$\simeq=0=\simeq \simeq \cong$

으으으응ㅇㅇㅇㅇㅡ

$\times \times \times \times \times \times \times$

드요

$\therefore-\infty$

의은

으으으의

$\times \times \times \times \times \times \times$

$\infty$ 는 2 응

$\dot{r}-\dot{i} \dot{i}-\infty \infty$

$==\subseteq=\simeq \simeq \simeq$

으으응ㅇㅇㅇㅇㅇ응

$\times \times \times \times \times \times \times$

ㅈำำ

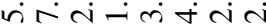

$\varrho \circ \simeq \simeq \simeq \simeq \simeq \simeq$

응ㅇㅇ응ㅇㅇㅇㅇㅇㅢ

$\times \times \times \times \times \times \times$

궁ํํำ

+ंतr

$=\because=\exists \simeq \simeq \simeq$

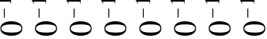

$\times \frac{1}{\times} \times \frac{1}{\times} \times \frac{1}{\times} \times$

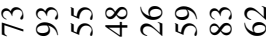

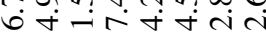

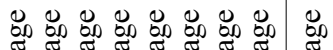

int n ก่ 


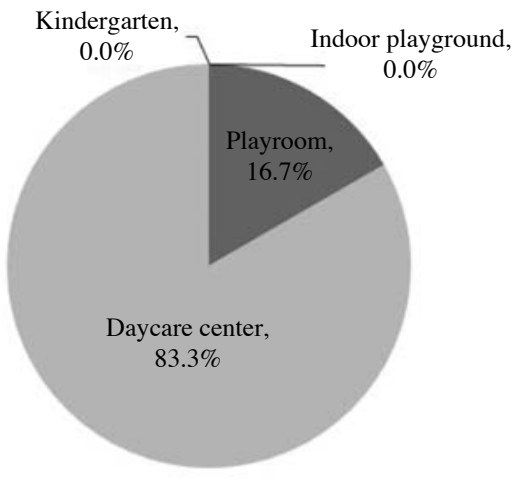

(a) Inhalation

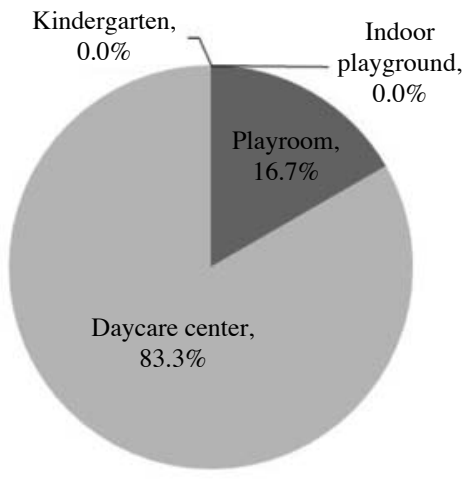

(b) Ingestion

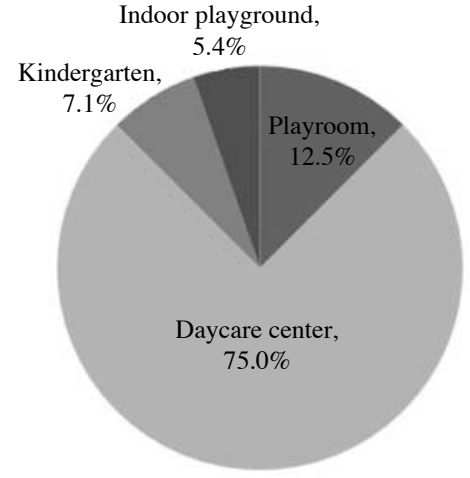

(c) Total exposure route

Fig. 3. Contribution of facility HI to the total HI of PBDEs for all facilities for 0.5 to 6-year-olds.

childcare facilities (playrooms and daycare centers), kindergartens and indoor playgrounds located in three cities, the selected facilities could not be said to represent all facilities. In particular, this study did not include data for free indoor playgrounds; we only examined those that charged admission fees. In addition, due to limitations in the cooperation of the investigated facilities, a sampling of representative facilities (area, year of construction, location, etc.) by population distribution rate could not be conducted. Moreover, not only PBDEs, but also trace metals, phthalates and pesticides were sampled in the surface wipe and hand wipe samples, and sufficient samples were difficult to secure. However, it was unclear whether or not the mass collected was dependent upon the surface area of the hands. Surface-area-normalized PBDE concentrations ranged from 3.3 to $1,970 \mathrm{pg} / \mathrm{cm}^{2}$ with a median value of $135 \mathrm{pg} / \mathrm{cm}^{2}$ (Heather et al., 2008). In addition, air and dust concentrations of PBDEs at indoor sites depend on how and where samples are collected. Further research is needed to evaluate the collection methods used in this study.

These limitations need to be supplemented through continuous future research. Nevertheless, our study is meaningful as a case study of a subject that remains largely unknown in South Korea. Although the average risk of exposure to PBDEs was within the safe range, they were detected continuously in the hand wipe, dust and surface wipe samples, indicating that indoor environmental risks should be managed through long-term monitoring.

\section{CONCLUSIONS}

This study evaluated the risks of multi-route exposure to PBDEs in childcare facilities (playrooms, day- care centers, kindergartens and indoor playgrounds). Assessment of multi-route exposure revealed that the average PBDE concentration was highest in indoor playgrounds $\left(1,440.33 \mathrm{pg} / \mathrm{m}^{3}\right)$, then kindergartens $\left(1,021.00 \mathrm{pg} / \mathrm{m}^{3}\right)$, followed by daycare centers $(789.21$ $\left.\mathrm{pg} / \mathrm{m}^{3}\right)$ and playrooms $\left(366.80 \mathrm{pg} / \mathrm{m}^{3}\right)$. The detection rate was $100 \%$. In the dust samples, PBDE concentration varied considerably from 560.95 to $32,829.88 \mathrm{ng} /$ g. In the air samples, the highest recorded concentration was $16,901.95 \mathrm{ng} / \mathrm{g}$ in indoor playgrounds. Of the eight isomers, the concentration of BDE-209 accounted for over $90 \%$ of the total PBDEs, which was similar to findings from previous studies.

The HI of non-carcinogenic toxicity of PBDEs was calculated by taking into consideration the behavioral characteristics of children in the facilities. The HI of the facilities did not exceed 0.1 . The $50^{\text {th }}$ percentile (median) $\mathrm{HI}$ was 0.0007 in playrooms, 0.001 in daycare centers, 0.0004 in kindergartens and less than 0.0001 in indoor playgrounds. Exposure rates to PBDEs were 71.4 to $96.1 \%$ by ingestion and 3.7 to $28.2 \%$ by inhalation exposure, making these two routes the primary exposure pathways.

Because PBDEs were continuously detected in all routes (indoor air, dust, surface wipe and hand wipe), the existence of pollutants was clearly confirmed. Although the risk was not high in these facilities, including childcare facilities, kindergartens and indoor playgrounds, further continuous monitoring and assessment of the risks of multiple exposures to PBDEs will be needed to prevent various diseases.

\section{ACKNOWLEDGEMENT}

This study was part of project supported by the Korean Ministry of Environment (Environmental Health 
Policy Division Office of Environmental Health), "Risk Assessment in Facilities for Child" ('07-'08), and we appreciate the support.

\section{REFERENCES}

Allen, J.G., Mcclean, M.D., Stapleton, H.M., Nelson, J.W., Webster, T.F. (2007) Personal Exposure to Polybrominated Diphenyl Ethers (PBDEs) in Residential Indoor Air. Environmental Science \& Technology 41, 45744579.

Allen, J.G., Mcclean, M.D., Stapleton, H.M., Webster, T.F. (2008) Critical factors in assessing exposure to PBDEs via house dust. Environment International 34, 1085-1091.

Birnbaum, L.S., Cohen Hubal, E.A. (2006) Polybrominated diphenyl ethers: a case study for using. biomonitoring data to address risk assessment questions. Environmental Health Perspectives 114(11), 1770-1775.

Birnbaum, L.S., Staskal, D.F. (2004) Brominated flame retardants: cause for concern?. Environmental Health Perspectives 112(1), 9-17.

Butte, W., Heinzow, B. (2002) Pollutants in house dust as indicators of indoor contamination, In Reviews of Environmental Contamination and Toxicology. SpringerVerlag, New York, pp. 175, 1-46.

Cetin, B., Odabasi, M. (2011) Polybrominated diphenyl ethers (PBDEs) in indoor and outdoor window organic films in Izmir, Turkey. Journal of Hazardous Materials 185, 784-791.

Colt, J.S., Zahm, S.H., Camann, D.E., Hartge, P. (1998) Comparison of pesticides and other compounds in carpet dust samples collected from used vacuum cleaner bags and from a high volume surface sampler. Environmental Health Perspectives 106(11), 721-724.

EEC (1996) European workshop on the impact of endocrine disrupters on human health and wildlife. Weybridge: UK.

Eriksson, P., Jakobsson, E., Fredriksson, A. (2001) Brominated flame retardants: a novel class of developmental neurotoxicants in our environment?. Environmental Health Perspectives 109(9), 903-908.

Gevao, B., Al-Bahloul, M., Al-Ghadban, A.N., Ali, L., AlOmair, A., Helaleh, M., Al-Matrouk, K., Zafar, J. (2006) Polybrominated diphenyl ethers in indoor air in Kuwait: Implications for human exposure. Atmospheric Environment 40, 1419-1426.

Gordon, S.M., Callahan, P.J., Nishioka, M.G., Brinkman, M.C., O'Rourke, M.K., Lebowitz, M.D., Moschandreas, M.J. (1999) Residential environmental measurements in the National Human Exposure Assessment Survey (NHEXAS) pilot study in Arizona: preliminary results for pesticides and VOCs. Journal of Exposure Analysis and Environmental Epidemiology 9(5), 456470.

Harrad, S., Ibarra, C., Diamond, M., Melymuk, L., Rob- son, M., Douwes, J., Roosens, L., Dirtu, A.C., Covaci, A. (2008) Polybrominated diphenyl ethers in domestic indoor dust from Canada, New Zealand, United Kingdom and Unites States. Environment International 34, 232-238.

Hazrati, S., Harrad, S. (2006) Causes of variability in concentrations of Polychlorinated Biphenyls and Polybrominated Diphenyl Ethers in indoor air. Environmental Science \& Technology 40, 7584-7589.

Health Canada (2004) State of the Science Report for a Screening Health Assessment Polybrominated Diphenyl Ethers (PBDEs) [Tetra-, Penta-, Hexa-, Hepta-, Octa-, Nona- and Deca- Congeners], Minister of Health- Health Canada.

Heather, M.S., Shannon, M.K., Joseph, G.A., Michael, D.M., Thomas, F.W. (2008) Measurement of Polybrominated Diphenyl Ethers on Hand Wipes: Estimatng Exposure from Hand-to-Mouth Contact. Environmental Science \& Technology 42, 3329-3334.

Jang, J.Y., Jo, S.N., Kim, S.Y., Kim, S.J., Cheong, H.K. (2007) Korean exposure factors handbook (KEFH). Korea Ministry of Environment, Seoul.

Kang, Y., Wang, H.S., Cheung, K.C., Wong, M.H. (2011) Polybrominated diphenyl ethers (PBDEs) in indoor dust and human hair. Atmospheric Environment 45, 2386-2393.

Lioy, P.J., Freeman, N.C.G., Millette, J.R. (2002) Dust: A metric for use in residential and building exposure assessment and source characterization. Environmental Health Perspectives 110(10), 969-983.

Mandalakis, M., Atsarou, V., Stephanou, E.G. (2008) Airborne PBDEs in specialized occupational settings, houses and outdoor urban areas in Greece. Environmental Pollution 155, 375-382.

NIER (National Institute of Environmental Research) (2007) Assessment of exposure and risk by Children's product (I): focused on oral exposure.

Schecter, A., Paepke, O., Joseph, J.E., Tung, K. (2005) Polybrominated diphenyl ethers (PBDEs) in US computers and domestic carpet vacuuming: possible sources of human exposure. Journal of Toxicology and Environmental Health 68, 501-513.

Stapleton, H.M., Dodder, N.G., Offenberg, J.H., Schantz, M.M., Wise, S.A. (2005) Polybrominated diphenyl ethers in house dust and clothes dryer lint. Environmental Science \& Technology 39(4), 925-931.

Stapleton, H.M., Dodder, N.G. (2008) Photodegradation of decabromodiphenyl ether (BDE 209) in house dust by natural sunlight. Environmental Toxicology and chemistry 27(2), 306-312.

Tan, J., Cheng, S.M., Loganath, A., Chong, Y.S., Obbard, J.P. (2007) Polybrominated diphenyl ethers in house dust in Singapore. Chemosphere 66(6), 985-992.

Toms, L.M.L., Hearn, L., Kennedy, K., Harden, F., Bartkow, M., Temme, C., Mueller, J.F. (2009) Concentrations of polybrominated diphenyl ethers (PBDEs) in matched samples of human milk, dust and indoor air. Environment International 35, 864-869. 
Umweltbundesamt (2000) Erarbeitung von Bewertungsgrundlagen zur Substitution umweltrelevanter Flammschutzmittel, Texte 25, vol. I-III, Berlin.

US EPA (US Environmental Protection Agency) (2002) Child Specific Exposure Factors Handbook (CEFH), United States Environmental Protection Agency, Washington DC.

US EPA (2007) Brominated diphenyl ethers in water soil, sediment and tissue by HRGC/HRMS, United States Environmental Protection Agency, Washington DC. Available from: <http://www.epa.gov/waterscience/ methods/method/files/1614.pdf>

US EPA (2008a) Toxicological Review of 2,2',3,3',4,4', $5,5^{\prime}, 6,6^{\prime}$-Decabromodiphenyl ether (BDE-209) in Support of Summary Information on the Integrated Risk Information System (IRIS), National Center for Environmental Assessment, Washington, DC. Available online at http://www.epa.gov/iris.

US EPA (2008b) Toxicological Review of 2,2',4,4',5,5'Hexabromodiphenyl ether (BDE-153) in Support of Summary Information on the Integrated Risk Information System (IRIS), National Center for Environmental Assessment, Washington, DC. Available online at http://www.epa.gov/iris.

US EPA (2008c) Toxicological Review of 2,2',4,4',5-Pentabromodiphenyl ether (BDE-99) in Support of Summary Information on the Integrated Risk Information System (IRIS), National Center for Environmental Assessment, Washington, DC. Available online at http:// www.epa.gov/iris.

US EPA (2008d) Toxicological Review of 2,2',4,4' -Tetrabromodiphenyl Ether (BDE-47) in Support of Summary Information on the Integrated Risk Information System (IRIS), National Center for Environmental Assessment, Washington, DC. Available online at http:// www.epa.gov/iris.

Viberg, H., Frederiksson, A., Eriksson, P. (2003a) Neonatal exposure to polybrominated diphenyl ether (PBDE 153) disrupts spontaneous behaviour, impairs learning and memory, and decreases hippocampal cholinergic receptors in adult mice. Toxicology and Applied Phar- macology 192(2), 95-106.

Viberg, H., Fredriksson, A., Jakobsson, E., Om, U., Eriksson, P. (2003b) Neurobehavioral derangements in adult mice receiving decabromodiphenyl ether (PBDE 209) during a defined period of neonatal brain development. Toxicological Sciences 76, 112-120.

Viberg, H., Fredriksson, A., Eriksson, P. (2004a) Neonatal exposure to the brominated flame-retardant, 2,2', 4,4',5-pentabromodiphenyl ether, decreases cholinergic nicotinic receptors in hippocampus and affects spontaneous behaviour in the adult mouse. Environmental Toxicology and Pharmacology 17, 61-65.

Webster, T., Vieira, V., Schecter, A. (2005) Estimating exposure to PBDE-47 via air, food and dust using Monte Carlo methods. Organohalogen Compound 67, 505508.

Weil, E.D., Levchik, S.V. (2007) Flame retardants for polystyrenes in commercial use or development. Journal of Fire Sciences 25, 241-265.

Wilford, B., Shoeib, M., Harner, T., Zhu, J., Jones, K.C. (2005) Polybrominated diphenyl ethers in indoor dust in Ottawa, Canada: implications for sources and exposure. Environmental Science \& Technology 39, 70277035 .

Zartarian, V.G., Xue, J., Ozkaynak, H.A., Dang, W., Glen, G., Smith, L., Stallings, C.A. (2005) probabilistic exposure assessment for children who contact CCAtreated playsets and decks using the stochastic human exposure and dose simulation model for the wood preservative scenario (SHEDS-WOOD). Final Report. EPA/600/X-05/009. Washington DC, U.S. Environmental Protection Agency. Available from:URL:http:// www.epa.gov/heasd/sheds/caa_treated.htm.

Zhao, D., Little, J.C., Cox, S.S. (2004) Characterizing polyurethane foam as a sink for or source of volatile organic compounds in indoor air. Journal of Environmental Engineering 130, 983-989.

(Received 27 March 2011, revised 22 October 2011 accepted 22 October 2011) 